UDK 528.7

\title{
NUMERICAL EVALUATION AND APPLICATION-ORIENTED ANALYSIS FOR FORWARD AND INVERSE RATIONAL FUNCTION MODELS OF TERRAIN-INDEPENDENT CASE IN SATELLITE IMAGERY
}

\author{
Sorosh JAHANDIDEH ${ }^{1}$, Ali AZIZI ${ }^{2}$, Nasser NAJIBI ${ }^{3}$ \\ 1, 2 Department of Surveying and Geomatics Engineering, Faculty of Engineering, \\ University of Tehran, Iran \\ ${ }^{3}$ Shanghai Astronomical Observatory, Chinese Academy of Sciences, Shanghai, China \\ E-mails: ${ }^{1}$ sjahandideh@ut.ac.ir; ${ }^{3}$ nsr.najibi@gmail.com (corresponding author)
}

Received 25 June 2014; accepted 9 September 2014

\begin{abstract}
Terrain-independent Rational Polynomial Coefficients (RPCs) are considered as most important part of the optical satellite and aerial imagery data processing especially those ones with high resolution since the proposed RPCs by the aerospace companies have some limitations in particular for using directly by the geoscientists in environmental studies and other Earth observation applications. While the inverse RPCs have more advantageous rather than direct ones, in this study, a new approach is presented in order to provide the inverse RPCs from direct ones and also to satisfy satellite imagery products users. In order to do this, first a spatial 3D-cubic is going to be fitted to the study area approximately including necessary altimetry layers numbers. Next, a range of virtual control points are being created in those altimetry layers randomly and then these points are going to be shifted to the image space by means of given direct RPCs. Hence, the inverse RPCs computes from the direct ones by space resection technique. Finally, the ground coordinates for the corresponding points have derived from different space intersection methodologies, direct RPCs and also inverse ones. Moreover, comparative tests have been developed to assess the effects of different altimetry layers numbers and also the number of virtual control points on the quality of derived inverse RPCs. It is demonstrated here that the precision of derived RPCs are increasing as much as the number of altimetry layers and control points increase. The proposed methodology, computations, data processing and results evaluation are discussed in details.
\end{abstract}

Keywords: Forward/Inverse RPCs, Rational function, Digital terrain model, Ortho-photo, High-resolution optical image.

\section{Introduction}

Prior to the use of rational functions in military applications and facilities, the physical equations and models such as coplinearity equation were using to transfer from object space coordinates to image space ones (Sohn et al. 2005; Lin, Yuan 2008; Najibi, Abedini 2010). It is needless to say that Earth surface studies are very critical in these days (e.g. Sarabandi et al. 2008; Najibi, Jin 2013; Najibi, Arabsheibani 2013; Najibi et al. 2013), however, this demand asks for more sophisticated Earth observations sensors and other useful imagery applications especially for environment studies to create new advanced method (Tao et al. 2004; Najibi, Abedini 2013) and also open up the rational functions field, but the researchers did not get into details about them (Tao, Hu 2002). The rational functions were extremely used with the launch of first high-resolution imagery satellites. Thus, using this model on three-dimensional (3D) modelling software became a common approach (Madani 1999), although the geoscientists still did not achieve a complete model and information. According to this, the initial studies to assess the possibility of using rational functions instead of the physical functions and comprehensive study of them were done from aerial imagery and photogrammetry view (Dowman, Dolloff 2000; Li et al. 2009). This inevitably opened a new interesting field for further studies and experiments. Although, Tao and $\mathrm{Hu}$ (2001) 
studied rational functions in independent case and dependent on ground state, but this study was done mostly based on numerical tests rather than quality approaches and also focused mostly on forward and inverse rational functions for 3D modelling comprehensively. This study also improved the spatial intersection with inverse rational functions. The evaluation of IKONOS imagery is done from aspect of both radiometric and geometric by Baltsavias et al. (2001). Moreover, Grodechi and Dial (2003) studied the block adjustment of high-resolution images using rational functions. Besides, Samadzadegan et al. (2005) mentioned a new method to optimize the rational functions coefficients. Additionally, Chen et al. (2006) evaluated geometric performance of rational functions in order to be replaced by Rigor's models. The geometric assessments of satellite imagery (e.g. QuickBird) are being discussed in details by Aguilar et al. (2007 and 2008). Furthermore, Habib et al. (2007) compared geometric accuracy of rational functions with general and Rigors models for satellite and aerial images. Zhang et al. (2011) studied the possibility of using rational functions instead of Rigors in Synthetic Aperture Radar (SAR) images. Recently Wang et al. (2014) studied the geometric accuracy validation for ZY-3 satellite imagery in order to investigate the principle of on-orbit calibration and production processes of its sensor-corrected products.

Terrain-independent Rational Polynomial Coefficients (RPCs) are considered as essential segments of the high-resolution optical satellite images due to the fact that they are generated by the aerospace organizations through fitting Rational Function Models (RFMs) to the physical model. The generated terrain coordinates by RPCs can be reached a reasonable level of geometric accuracy with just a single ground control point which means that the direct georeferencing is almost feasible with the supplied RPCs. However, the aerospace organizations and companies conventionally use the forward RFM to generate RPCs, but the RPCs can also be generated by the inverse RFM. Now, the question arises as to which of the above cases (direct coefficients or inverse ones) are computationally more efficient for various applications? This question has not been answered so far properly. Therefore, the main objective of this paper is to analyse the computational efficiency of the forward and inverse RFMs and also to introduce optimized models associated with different applications of these functions. The optimum choice of the inverse or forward RFMs plays an important role for those applications that require real-time aerial imagery processing products. The main applications of terrainindependent rational functions can be summarized in the following three categories as:

1) Digital elevation data generation,

2) Ortho-rectification,

3) Transformation of pixels to $3 D$ object space (mono-plotting).

Although the pixel coordinates in forward RFM are expressed as a function of their corresponding ground coordinates, whereas the ground coordinates with the inverse RFM are the functions of their pixel coordinates. Thus, for those applications in which the transformation takes place from the image space to the object space (e.g. mono-plotting), the solution of the forward RFM (using Newton-Raphson approach) requires the linearization of all three components of the ground coordinates $(X, Y, Z)$ and consequently the initial approximations for all three components are required. Moreover, the loop structure also becomes complicated. However, the inverse RFM for the solution of the same problem requires only linearization with respect to the $Z$ component with a simple loop structure. Therefore, for applications in which the transformation is performed from the image space to the object space, inverse rational equations will be computationally more efficient with a larger radius of convergence. Conversely, for those applications in which the transformation is performed from the object space to the image space (e.g. ortho-rectification), the inverse RFM is not an optimum solution since linearization is carried out with respect to the pixel coordinates $x, y$ and their corresponding $Z$ component. In this case, solving the problem by using forward function would be more efficient.

In this paper, the forward and inverse RFM algorithms are implemented and a numerical analysis is conducted for different applications using stereo Cartosat-1 satellite images over a highly mountainous terrain. Since the aerospace organizations customarily generate forward RPCs, The application-oriented optimum solution requires an algorithm for the conversion of the forward RPCs to the inverse RPCs. This paper also proposes a forward model to inverse RPC conversion algorithm. The numerical evaluation conducted in this paper indicates that with the implemented algorithm no significant loss of precision is occurred during the conversion process and as far as the precision of the generated object coordinates is concerned, the converted inverse RPCs are practically equivalent to the forward RPCs. Based on this; Figures 1 and 2 present step-by-step the instructions and 
also the flowchart of the proposed method respectively. In Figures 1 and 2, the mentioned steps including the creating of the control points, data processing and results evaluation can be seen which in are going to describe as the following sections:

- The first step; creating the control points: in first stage, the imagery area's altitude and planimetric range is approximately determined. Then, the cube is fitted to this imagery area by using a specified range. Next, the classified planimetric area is girding and also a specified altimetry range is divided into given number of layers. At each layer, the specified number of virtual control point is created randomly.

- The second step; the data processing: at first stage, virtual control points are transferred from object space to image space by use of forward rational functions (those coefficients which have been received from aerospace organizations). Moreover, the pixel coordinates of each point on the image surface is obtained. Then, the space resection is calculated using the inverse rational functions. This process is done for both backward and forward images. Since the inverse rational coefficients of each image and pixel coordinates of each virtual control points are available, it is possible to do the space intersection for quality control of the inverse rational function coefficients. Furthermore, it is straightforwardly to be done the direct comparisons between the intersection with forward rational functions and also inverse rational function, as well as the intersection with forward rational function.

- The third step; the results evaluation: Those factors which are affecting the quality of the produced inverse rational coefficients from forward rational coefficients such as number of virtual control points and altimetry layers are being tested. Moreover, the relationship between the space intersection initialized values and convergence speed and error rates are being assessed.

In the following sections, first a brief introduction to the rational functions is given besides they are assessed based on terrain dependent and also independent. Then, the direct rational functions and inverse ones as well as space resection and intersection by using inverse rational functions are described in details and eventually the quality of derived inverse rational functions coefficients from direct rational ones as well as the precision of concluded 3D coordinates are discussed and expatiated completely.

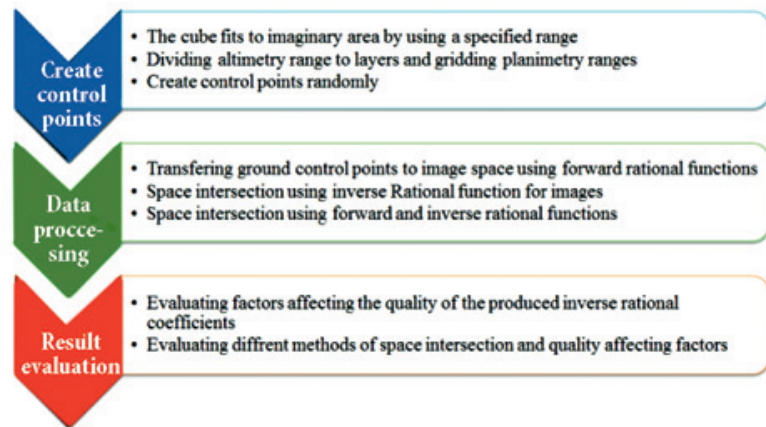

Fig. 1.Step-by-step instructions of proposed method for conversion of forward RPCs to the inverse ones

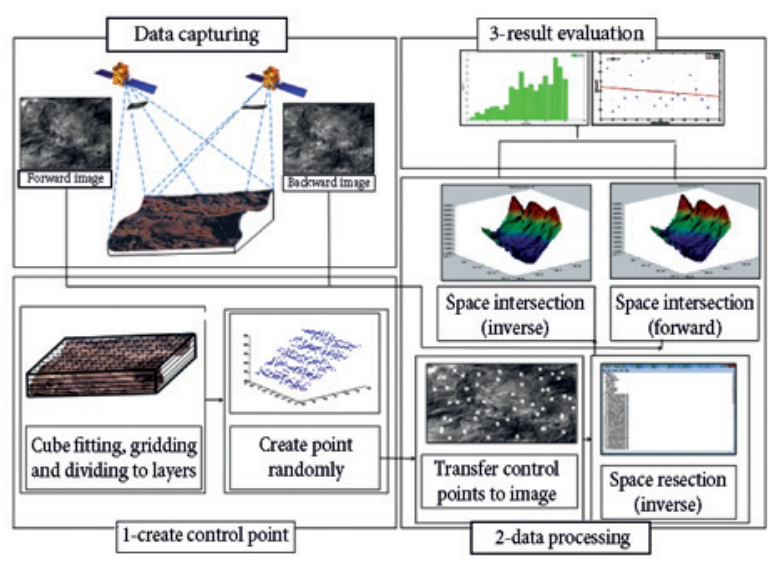

Fig. 2. The flowchart of proposed method for conversion of forward RPCs to the inverse ones

\section{Materials and methods}

The Rational Function Model (RFM) are mathematical functions which are very similar to the projective equations (Madani 1999). Rational functions are employed to communicate between the two-dimensional (2D) image coordinates $(r, c)$ and 3D ground coordinates $(X, Y, Z)$ and vice versa. The functions of the coordinates $(X, Y)$ as a ratio of polynomials $(r, c, Z)$ which can be as total of 78 parameters, to establish the relationship between image space and ground space rational equations are presented as follows (Tao, Hu 2001):

$$
\begin{aligned}
& Y=\frac{P 3(r, c, Z)}{P 4(r, c, Z)}, \\
& X=\frac{P 1(r, c, Z)}{P 2(r, c, Z)} .
\end{aligned}
$$

The rational function coefficients are going to be solved using one of the terrain dependent or independent methods which are related to existing or lack of satellite physical parameters respectively at imagery time. 
However, the terrain independent method will be employed when the satellite physical parameters are available and or there is not any ground control information. In this case, by designing a 3D terrestrial network and corresponding image network, the coefficients of the model equations are obtained directly. This is common method for obtaining the rational coefficients because it does not require to define the ground control points. Based on this, the rational function coefficients which are going to be obtained using this method will be greatly affected by accuracy of the satellite physical parameters and that assumed terrestrial network precision (Tao, Hu 2001).

Conversely, the terrain dependent method will be applied when the satellite physical parameters are not available. In this case, there is a requirement to have the ground control points. Hence, the ground control points for this method can be achieved by using the present conventional methods. Although in this scenario, the accuracy of ground control points will play a key role and thus the rational functions is highly dependent on the accuracy of these control points.

Those rational functions that transfer the points coordinates from ground space to the image one can be split into two equations:

- Forward equations;

- Inverse equations.

Each one of these equations would lead to another one which is dependent on the type of used inquiry. In the following sections, they will be discussed in details.

Basically the forward models are being used to transfer from ground space to image one. The rational functions are discussed as a function of the ground points coordinates as:

$r_{n}=\frac{P 1\left(X_{n}, Y_{n}, Z_{n}\right)}{P 2\left(X_{n}, Y_{n}, Z_{n}\right)}=\frac{\sum_{i=0}^{m 1} \sum_{j=0}^{m 2} \sum_{k=0}^{m 3} a_{i j k} X_{n}^{i} Y_{n}^{j} Z_{n}^{k}}{\sum_{i=0}^{n 1} \sum_{j=0}^{n 2} \sum_{k=0}^{n 3} b_{i j k} X_{n}^{i} Y_{n}^{j} Z_{n}^{k}}$,

$c_{n}=\frac{P 3\left(X_{n}, Y_{n}, Z_{n}\right)}{P 4\left(X_{n}, Y_{n}, Z_{n}\right)}=\frac{\sum_{i=0}^{m 1} \sum_{j=0}^{m 2} \sum_{k=0}^{m 3} c_{i j k} X_{n}^{i} Y_{n}^{j} Z_{n}^{k}}{\sum_{i=0}^{n 1} \sum_{j=0}^{n 2} \sum_{k=0}^{n 3} d_{i j k} X_{n}^{i} Y_{n}^{j} Z_{n}^{k}}$.

In order to reduce the computational error, the ground and image coordinates are normalized in the range between $(-1,1)$ (Cox 2010). Accordingly, $r_{n}$ and $c_{n}$ are the normalized coordinate values in the form of rows and columns pixels in the image space and also
$X_{n}, Y_{n}$ and $Z_{n}$ are normalized values of ground space coordinates. Moreover, $a_{i j k}, b_{i j k}, c_{i j k}$ and $d_{i j k}$ are the polynomial coefficients that are discussed as RPCs. $m_{1}$, $m_{2}$ and $m_{3}$ are maximum powers of ground coordinates and are typically 3 . It is good to note that the total power of each polynomial term is determined to be at maximum 3. The numerator and denominator of rational functions that are 3-degree polynomial equations consist of 20 sentences as given:

$$
\begin{aligned}
& P=\sum_{i=0}^{m 1} \sum_{j=0}^{m 2} \sum_{k=0}^{m 3} a_{i j k} X^{i} Y^{j} Z^{k}=a_{0}+a_{1} Z+a_{2} Y+a_{3} X+ \\
& a_{4} Z Y+a_{5} Z Y+a_{6} Y X+a_{7} Z^{2}+a_{8} Y^{2}+a_{9} X^{2}+a_{10} Z Y X+ \\
& a_{11} Z^{2} Y+a_{12} Z^{2} X+a_{13} Y^{2} Z+a_{14} Y^{2} X+a_{15} Z X^{2}+ \\
& a_{16} Y X^{2}+a_{17} Z^{3}+a_{18} Y^{3}+a_{19} X^{3}
\end{aligned}
$$

To normalize the ground and pixel coordinates, the following equations are used as:

$$
\begin{aligned}
& c_{n}=\frac{c-c_{0}}{c_{s}}, \\
& r_{n}=\frac{r-r_{0}}{r_{s}}, \\
& X_{n}=\frac{X-X_{0}}{X_{s}}, \\
& Y_{n}=\frac{Y-Y_{0}}{Y_{s}}, \\
& Z_{n}=\frac{Z-Z_{0}}{Z_{s}} .
\end{aligned}
$$

In above equations, $\mathrm{r}_{0}$ and $c_{0}$ are image coordinates displacement values and $r_{s}$ and $c_{s}$ are scale numbers of the image coordinates. Similarly, $X_{0}, Y_{0}$ and $Z_{0}$ are ground coordinates displacement values and also $X_{s}, Y_{s}$ and $Z_{s}$ are scale numbers for ground coordinates.

The inverse model of equations is described as the reverse of forward equation which will be used to transfer from the image space to ground one directly. The rational functions are discussed as follows:

$$
Y_{n}=\frac{P 3\left(r_{n}, c_{n}, Z_{n}\right)}{P 4\left(r_{n}, c_{n}, Z_{n}\right)}=\frac{\sum_{i=0}^{m 1} \sum_{j=0}^{m 2} \sum_{k=0}^{m 3} c_{i j k} r_{n}^{i} c_{n}^{j} Z_{n}^{k}}{\sum_{i=0}^{n 1} \sum_{j=0}^{n 2} \sum_{k=0}^{n 3} d_{i j k} r_{n}^{i} c_{n}^{j} Z_{n}^{k}},
$$

$$
X_{n}=\frac{P 1\left(r_{n}, c_{n}, Z_{n}\right)}{P 2\left(r_{n}, c_{n}, Z_{n}\right)}=\frac{\sum_{i=0}^{m 1} \sum_{j=0}^{m 2} \sum_{k=0}^{m 3} a_{i j k} r_{n}^{i} c_{n}^{j} Z_{n}^{k}}{\sum_{i=0}^{n 1} \sum_{j=0}^{n 2} \sum_{k=0}^{n 3} b_{i j k} r_{n}^{i} c_{n}^{j} Z_{n}^{k}},
$$


where the existing parameters and constants within above equations are being introduced and defined earlier in above sections.

The purpose of solving space resection using inverse rational functions is to obtain coefficient of these functions. The space resection using rational functions can be solved by direct methods and iterative method.

First, equation (11) and (12) can be rewritten as follows:

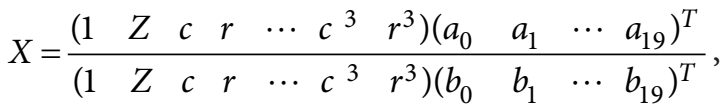

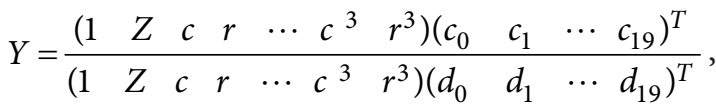

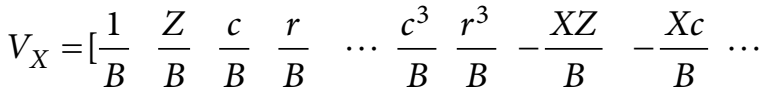

$$
\begin{aligned}
& \left.-\frac{X c^{3}}{B}-\frac{X r^{3}}{B}\right] . J-\frac{X}{B} \text {, }
\end{aligned}
$$

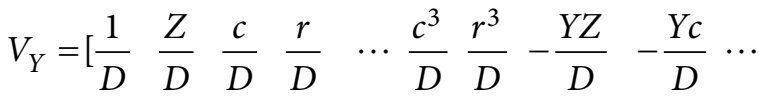

$$
\begin{aligned}
& \left.-\frac{Y c^{3}}{D}-\frac{Y r^{3}}{D}\right] \cdot k-\frac{Y}{D}, \\
& B=\left(\begin{array}{llllllll}
1 & Z & c & r & \cdots & c^{3} & r^{3}
\end{array}\right) \cdot\left(\begin{array}{llll}
1 & b_{1} & \cdots & b_{19}
\end{array}\right)^{T}, \\
& J=\left(\begin{array}{llllllll}
a_{0} & a_{1} & \cdots & a_{19} & b_{0} & b_{1} & \cdots & b_{19}
\end{array}\right)^{T},
\end{aligned}
$$

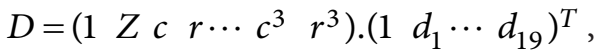

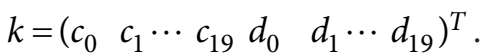

By considering $n$ control points with defined ground and image coordinate, the matrix equation (15) is created as follows:

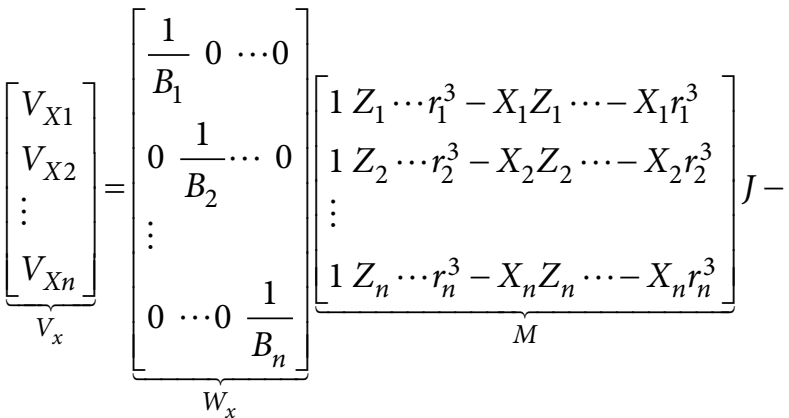

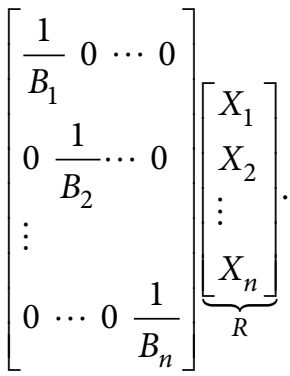

Then, it can be shown by simplicity as:

$$
V_{X}=W_{X} M J-W_{X} R \text {. }
$$

Moreover, the used normal equation has the form as follows:

$$
M^{T} W_{X}^{2} M J-M^{T} W_{X}^{2} M R=0 .
$$

In direct solution, the weight matrix is considered to be a unit matrix. According to this, the equation (15) can be solved as follows:

$$
J=\left(M^{T} M\right)^{-1} M^{T} R .
$$

Although equation (16) can be solved based on equation (15), but these two equations can be even solved simultaneously since there is no dependency between them and also they do not have any influence on each other. Based on this, the following equations can be derived as:

$$
\begin{aligned}
\underbrace{\left[\begin{array}{l}
V_{X} \\
V_{Y}
\end{array}\right]}_{V} & =\underbrace{\left[\begin{array}{cc}
W_{X} & 0 \\
0 & W_{Y}
\end{array}\right]}_{W} \underbrace{\left[\begin{array}{cc}
M & 0 \\
0 & N
\end{array}\right]}_{T} \underbrace{\left[\begin{array}{l}
J \\
K
\end{array}\right]}_{I}-\left[\begin{array}{cc}
W_{X} & 0 \\
0 & W_{Y}
\end{array}\right] \underbrace{\left[\begin{array}{l}
R \\
C
\end{array}\right],}_{G} \\
V & =W T I-W G .
\end{aligned}
$$

The normal equation is defined to be evaluated as follow:

$$
T^{T} W^{2} T I-T^{T} W^{2} G=0 .
$$

If those available control and tie points are not distributed homogenously over entire evaluated area, it is necessary to apply the normal equation regularization. Since the mentioned reason makes the T-matrix as a rank deficient matrix which will result in lack of convergence in iterative solution. In order to solve the mentioned normal equation (27), the regularization technique is used here. Accordingly, the equation (27) can be written as follows (Tao, Hu 2001):

$$
\left(T^{T} W^{2} T+h^{2} E\right) I-T^{T} W^{2} G=0,
$$

where $E$ is the unit matrix. Generally the regularization parameter determination is difficult and a very complicated procedure; but here this value is set to 0.004 (Tao, Hu 2001). In order to solve it, the Tikhonov method is used as follows (Neumaier 1998):

$$
\begin{aligned}
& I_{(0)}=0, \\
& W_{(0)}=W\left(I_{(0)}\right)=E, \\
& I_{(s)}=I_{(s-1)}+\left(T^{T} W_{s-1}^{2} T+h^{2} E\right)^{-1} T^{T} W_{s-1}^{2} V_{(s-1)} .
\end{aligned}
$$

The coefficients of rational functions for two images are calculated by using space resection as mentioned above. In next step, by the existing of 
corresponding image coordinates in two images for points and the coefficients of the rational equations, the ground points coordinates can be calculated using the iterative space intersection of rational functions. For this case, three methods are described as follows:

In first method of solution, rational equations of direct $X$ and $Y$ can be extended to $Z$ using Taylor's method. In first approximation, the following equation is obtained as (Tao, $\mathrm{Hu} 2002$ ):

$$
\begin{aligned}
& X \approx \hat{X}+\frac{\partial X}{\partial Z} \Delta Z, \\
& Y \approx \hat{Y}+\frac{\partial Y}{\partial Z} \Delta Z,
\end{aligned}
$$

where in it:

$$
\begin{aligned}
& \frac{\partial X}{\partial Z}=\frac{\frac{\partial P 1}{\partial Z} P 2-P 1 \frac{\partial P 2}{\partial Z}}{P 2 P 2}, \\
& \frac{\partial Y}{\partial Z}=\frac{\frac{\partial P 3}{\partial Z} P 4-P 3 \frac{\partial P 4}{\partial Z}}{P 4 P 4}, \\
& \frac{\partial P}{\partial Z}=a_{1}+a_{4} c+a_{5} r+2 a_{7} Z+a_{10} c r+ \\
& 2 a_{11} c Z+2 a_{12} r Z+a_{13} c^{2}+a_{15} r^{2}+3 a_{17} Z^{2} .
\end{aligned}
$$

And value of $\hat{X}, \hat{Y}$ are obtained by placement of the approximate value of $r, c$ and $z$ in equation (11) and (12).

Using a pair of corresponding image points $\left(r_{1}, c_{1}\right)$ and $\left(r_{r}\right.$ and $\left.c_{r}\right)$, we have:

$$
\begin{aligned}
& X \approx \hat{X}_{l}+\frac{\partial X_{l}}{\partial Z} \Delta Z, \\
& Y \approx \hat{Y}_{l}+\frac{\partial Y_{l}}{\partial Z} \Delta Z, \\
& X \approx \hat{X}_{r}+\frac{\partial X_{r}}{\partial Z} \Delta Z, \\
& Y \approx \hat{Y}_{r}+\frac{\partial Y_{r}}{\partial Z} \Delta Z .
\end{aligned}
$$

By removing $X$ and $Y$, the error equations are obtained as follows:

$$
\begin{gathered}
V_{x}=\left(\frac{\partial X_{r}}{\partial Z}-\frac{\partial X_{l}}{\partial Z}\right) \Delta Z-\left(\hat{X}_{l}-\hat{X}_{r}\right), \\
V_{y}=\left(\frac{\partial Y_{r}}{\partial Z}-\frac{\partial Y_{l}}{\partial Z}\right) \Delta Z-\left(\hat{Y}_{l}-\hat{Y}_{r}\right),
\end{gathered}
$$

$$
\left[\begin{array}{c}
v_{X} \\
v_{Y}
\end{array}\right]=\left[\begin{array}{l}
\frac{\partial X_{r}}{\partial Z}-\frac{\partial X_{l}}{\partial Z} \\
\frac{\partial Y_{r}}{\partial Z}-\frac{\partial Y_{l}}{\partial Z}
\end{array}\right][\Delta Z]-\left[\begin{array}{c}
\hat{X}_{l}-\hat{X}_{r} \\
\hat{Y}_{l}-\hat{Y}_{r}
\end{array}\right] .
$$

Then by using least squares solution for above equation, it will be given as:

$$
\Delta Z=
$$

$$
\frac{\left(\hat{X}_{l}-\hat{X}_{r}\right) W_{X}\left(\frac{\partial X_{r}}{\partial Z}-\frac{\partial X_{l}}{\partial Z}\right)+\left(\hat{Y}_{l}-\hat{Y}_{r}\right) W_{Y}\left(\frac{\partial Y_{r}}{\partial Z}-\frac{\partial Y_{l}}{\partial Z}\right)}{W_{X}\left(\frac{\partial X_{r}}{\partial Z}-\frac{\partial X_{l}}{\partial Z}\right)^{2}+W_{Y}\left(\frac{\partial Y_{r}}{\partial Z}-\frac{\partial Y_{l}}{\partial Z}\right)^{2}} .
$$

$W_{X}$ and $W_{Y}$ are weight values of $X$ and $Y$.

In second method of solution, in a similar fashion to the above process, only that part of final equation to get $\Delta Z$, the function mentioned in Yang (2000) is being substituted as:

$$
\Delta Z=\frac{\left(\hat{X}_{l}-\hat{X}_{r}+\hat{Y}_{l}-\hat{Y}_{r}\right)}{\left(\frac{\partial X_{r}}{\partial Z}-\frac{\partial X_{l}}{\partial Z}+\frac{\partial Y_{r}}{\partial Z}-\frac{\partial Y_{l}}{\partial Z}\right)} .
$$

But in third method of solution, the linearized equation (37) and (39) are employed and put them equal to each other which will be led to equation (46) as follows:

$$
v_{X}=\left[\frac{\partial X_{r}}{\partial Z}-\frac{\partial X_{l}}{\partial Z}\right][\Delta Z]-\left[\hat{X}_{l}-\hat{X}_{r}\right] .
$$

From equation 46 , the $\Delta Z$ is eventually going to be obtained using least square solution method.

\section{Results and discussion}

Generally many various factors would affect the reliability and precision of inverse coefficients generated from forward coefficients such as the number of virtual control points and or the number of altimetry levels. Moreover, after providing the inverse rational coefficients, in the case of 3D coordinate's extraction for the tie points, we face three space intersection methods which the methods used for space intersection influence the final accuracy and the convergent speed of processing since various tests were conducted and followed. In these tests, the backward and forward satellite Cartosat-1 imagery for a very mountainous region was used.

The corresponding pair images are taken on August 13, 2007 for Rood-e-Hen (Iran) region. The terrestrial altitude difference is between 1300-3200 $\mathrm{m}$ and thus this area is considered as mountainous 


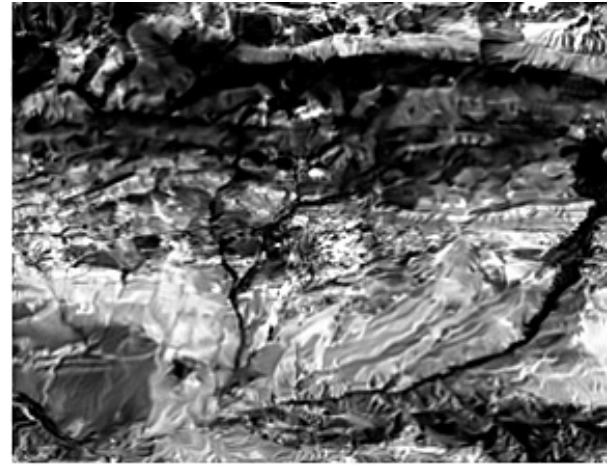

Fig. 3 . The study area imaged by Cartosat-1 satellite imagery

topology (Fig. 3). Although the initial rational coefficients are provided with aerospace organization, in order to investigate those factors which are affecting the accuracy of inverse rational coefficients generated from forward rational coefficients, the virtual control points were used. The virtual control points and 148 ground points are obtained using forward equations to check the accuracy of extracted 3D coordinates on the effects of initial values as well as the speed of the solution convergence (e.g. Fig. 3).

In order to assess the influence of altimetry layers on inverse rational functions coefficients quality, firstly, the imagery area's range should be specified. The cube fitted to the region and then it will be gridded with suitable interval. Next, 400 virtual control points for altimetry layers between 1 and 20 were created. The errors values were determined after processing which their rates decrease by increasing the number of altimetry layers. It is seen that quality of generated inverse rational coefficients in forward rational coefficients computation are improving while the number of altimetry layers are increasing (e.g. Figs 4-6).

Moreover, to analyze the influence of virtual control points numbers on inverse rational functions coefficients quality, the fitted cube is divided into 5 altimetry layers and gridded by the mentioned suitable interval. Then, for the virtual control points between 100 to 900 , the inverse rational coefficient generation process is done and the derived results were evaluated. It is seen that error is decreasing with increasing the number of virtual control points (e.g. Figs 7-9).

Furthermore, to investigate the influence of initial values on the space intersection precision, after obtaining the inverse rational coefficients and having the pixel coordinates of points in forward and backward images, we can calculate the 3D coordinates of the virtual control points by space intersection using inverse rational function (first method). In order to do this,

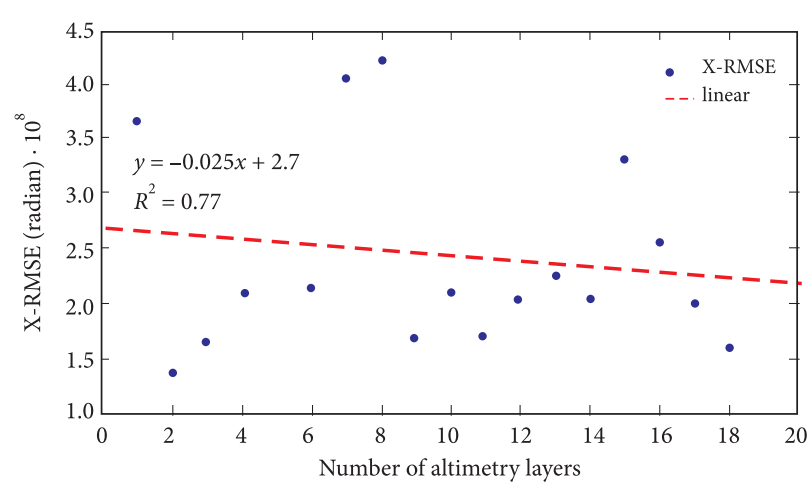

Fig. 4. RMSE (Longitude) changing rate by increasing altimetry layers

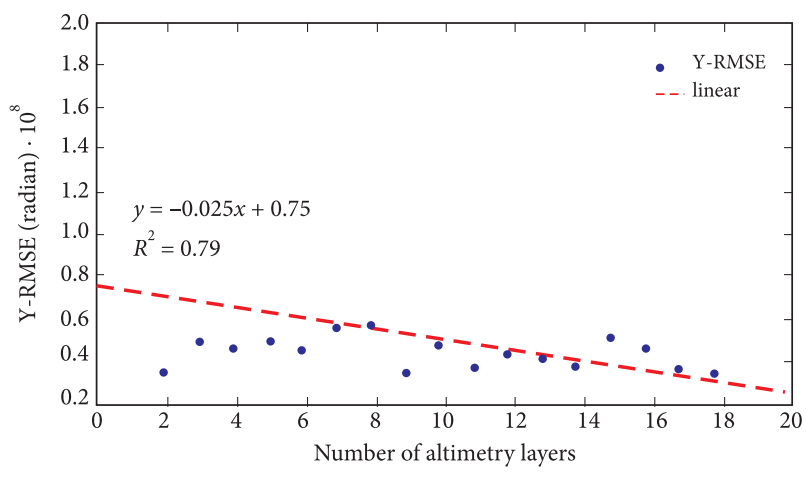

Fig. 5. RMSE (Latitude) changing rate by increasing altimetry layers

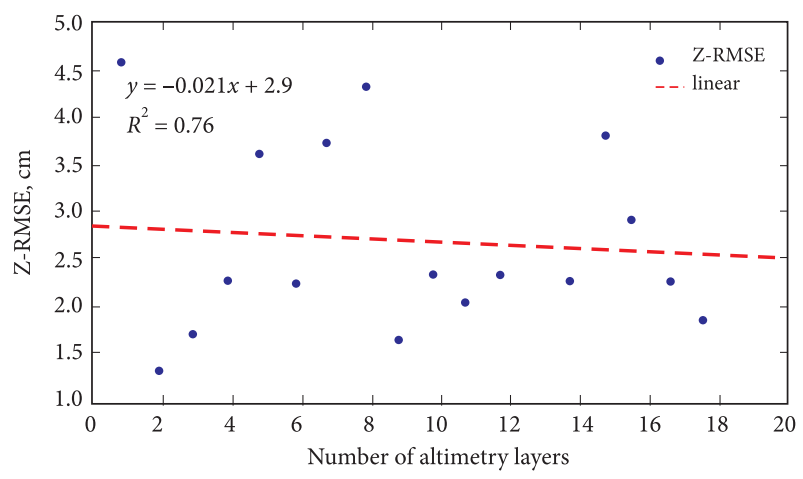

Fig. 6. RMSE (Height) changing rate by increasing altimetry layers

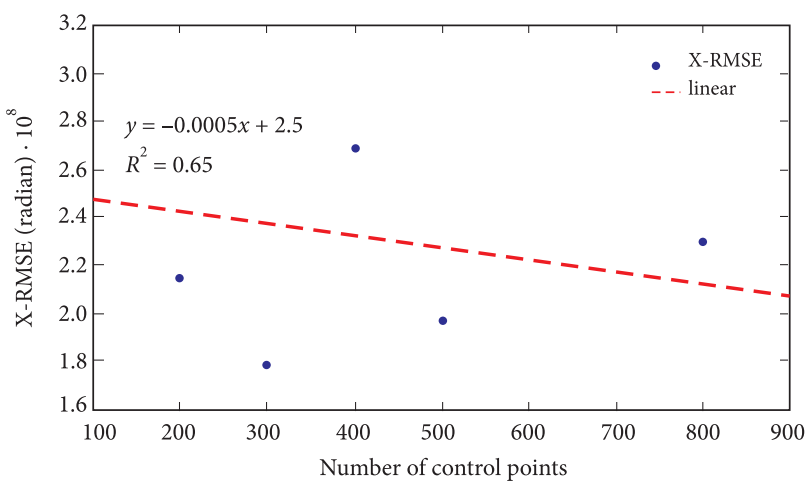

Fig. 7. RMSE (Longitude) changing rate by increasing control points 


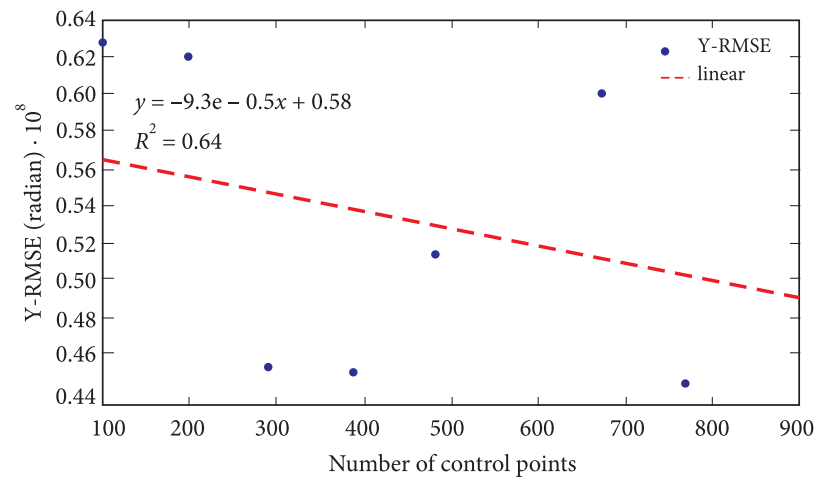

Fig. 8. RMSE (Latitude) changing rate by increasing control points

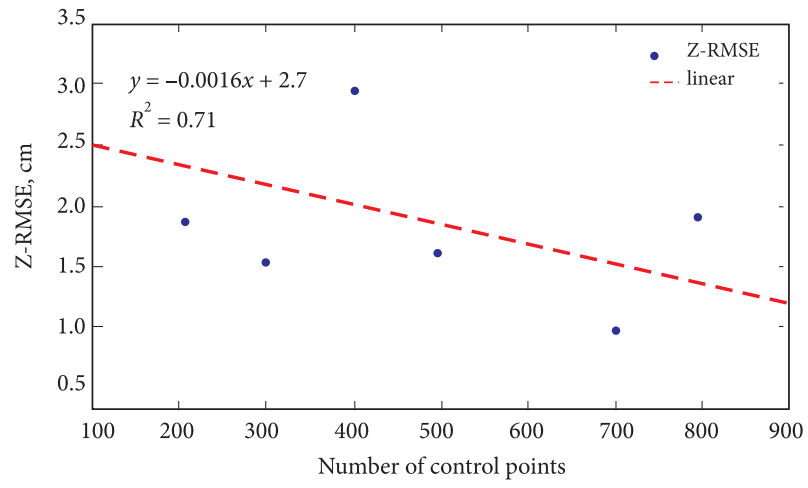

Fig. 9. RMSE (Height) changing rate by increasing control points

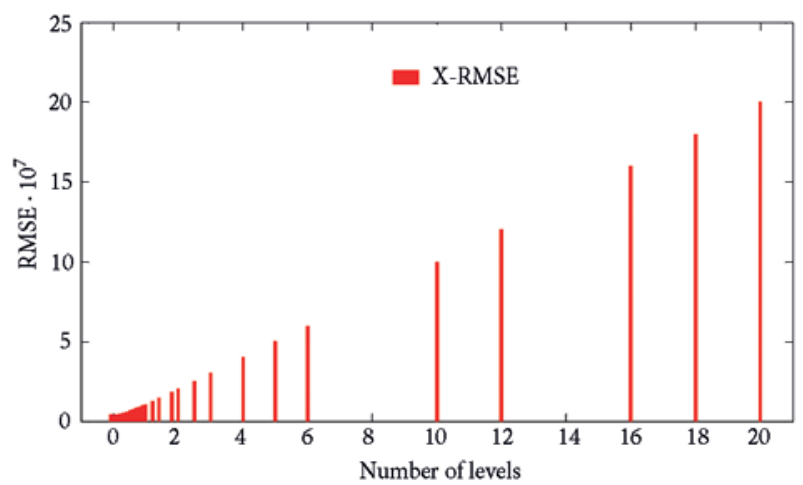

Fig.10. RMSE (Longitude) changing by increasing distance of initial value of point's height from real value of point's height

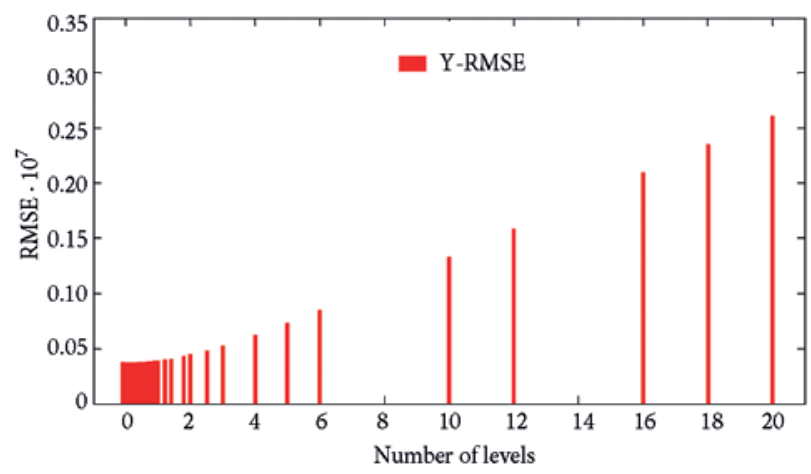

Fig. 11. RMSE (Latitude) changing by increasing distance of initial value of point's height from real value of point's height we need initial value of $Z$ for space intersection which is used in inverse rational functions. In this test, by differentiating the distance of the initial $Z$ value from real $Z$ one, the errors in points were calculated and evaluated. As it was expected, if the magnitude of the initial value is close to the actual one, the error rate is going to be reduced. Moreover, the error rate increases while the distance is increasing. Besides, the precision of result is acceptable by putting initial value of $Z$ equal to the average height of imagery area (e.g. Figs 10-12).

However, in order to assess the influence of iteration termination criterion on the space intersection precision, the inverse rational functions coefficients for forward and backward images were determined. The inverse space intersection (mentioned 3 methods) for virtual control points (400) with different iteration termination criterion processed by defining initial value of $Z$ equal to average height of imagery area and also the different termination criterion $\left(d Z \geq 10^{-a}\right)$ for iteration defined $(a, 0-7)$ are considered. As it can be seen (e.g. Figs 13-16), the precision is not going to be

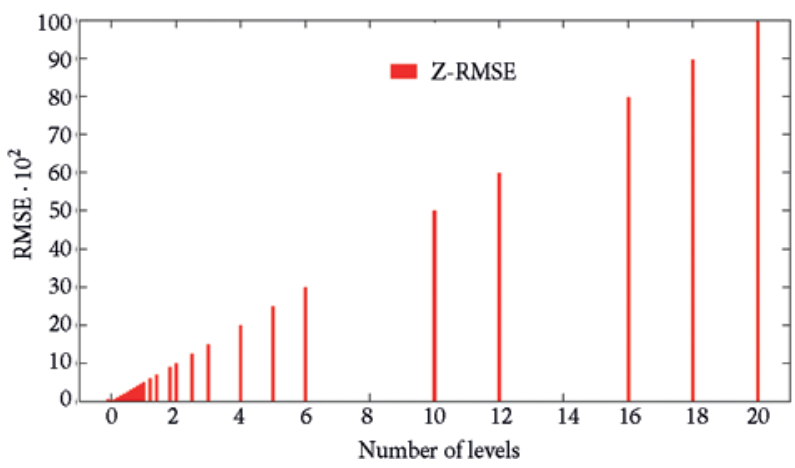

Fig. 12. RMSE (Height) changing by increasing distance of initial value of point's height from real value of point's height

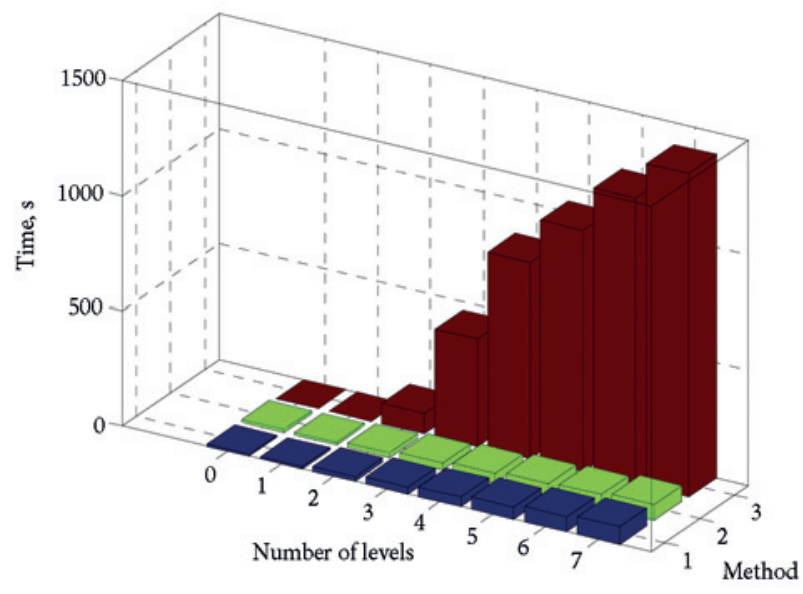

Fig. 13. Influence of iteration termination criterion on speed of convergence 
changed; conversely if a $\geq 4$, it reduces the speed of running the program.

Regarding the precision of forward intersection to inverse intersection, in this case, the space intersection (forward and inverse) is processed for those virtual control points (400) which had been created. The initial value for these points defined as the average height of imagery area with termination criterion defined $\left(d Z \leq 10^{-7}\right)$. It is demonstrated that the speed of program in forward intersection is faster but its precision in inverse intersection is better as it can be seen in Table 1 and also Figure 17.

Table 1.RMSE for different methods of intersection.

\begin{tabular}{|c|c|c|c|c|}
\hline & $\begin{array}{l}\text { X-RMSE } \\
\text { (radian) }\end{array}$ & $\begin{array}{l}\text { Y-RMSE } \\
\text { (radian) }\end{array}$ & $\begin{array}{l}\text { Z-RMSE } \\
(\mathrm{m})\end{array}$ & Time (s) \\
\hline 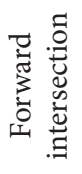 & 0.000000403 & 0.000000117 & 0.4902 & 0.5069 \\
\hline 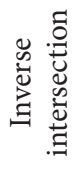 & 0.0000000152 & 0.0000000038 & 0.01511 & 203.0704 \\
\hline 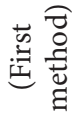 & 0.0000000139 & 0.0000000041 & 0.01494 & 69.729 \\
\hline 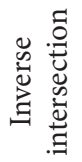 & 0.0000000139 & 0.00000000423 & 0.01495 & 2082.490 \\
\hline
\end{tabular}

\section{Conclusions}

As mentioned in the introduction, the aim of this study is to provide a mechanism for conversion of forward rational coefficients to the inverse ones. Regarding to this, the proposed methodology is based on the creation of virtual control points and ground ones independently. The first step will be to fit a cubic to the imagery area. Then, the virtual control points must be created in a cube. Also, the coordinates of the control points in image space should be calculated using rational coefficients. The inverse space resection is going to be applied for each of the employed satellite images. Finally, the inverse space intersection should be performed and thus the 3D model of imagery area will be created.

In this study, the practical statistical tests demonstrated that the proposed method can be handled when the inverse coefficients are available rather than forward coefficients. However, in order to maintain

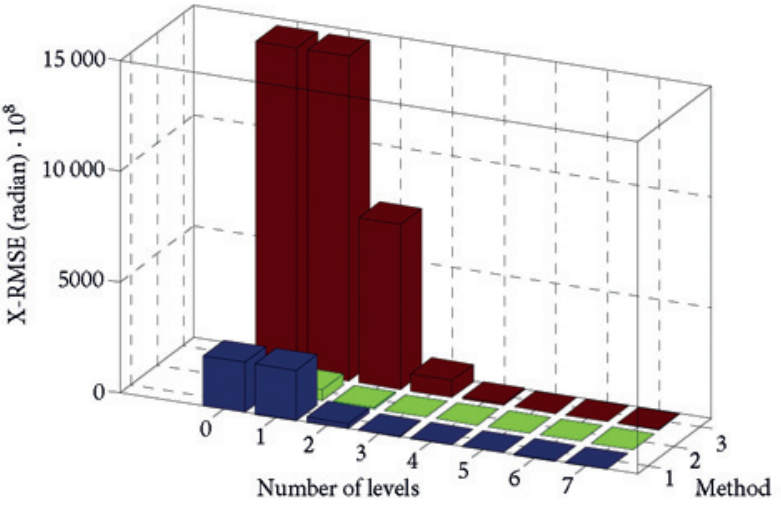

Fig. 14.RMSE (Longitude) changing by increasing termination criterion (a)

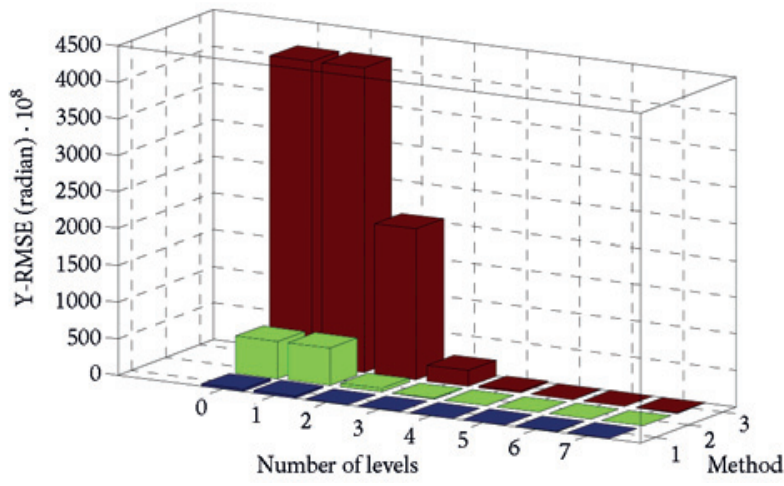

Fig. 15.RMSE (Latitude) changing by increasing termination criterion (a)

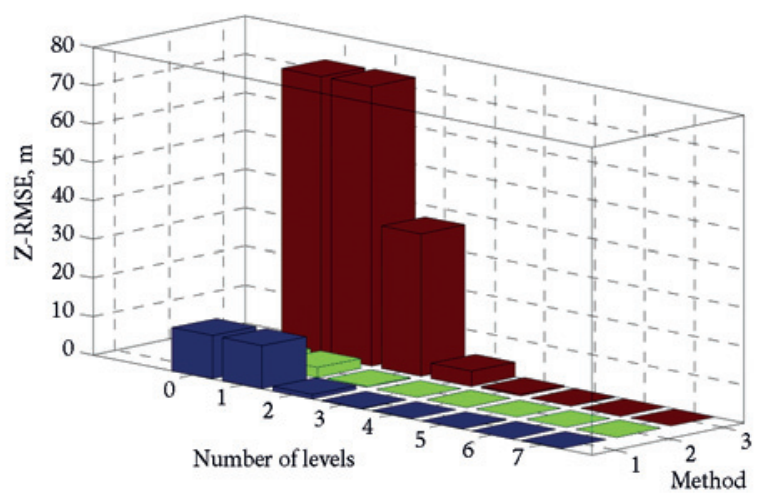

Fig. 16. RMSE (Height) changing by increasing $t$ ermination criterion (a)

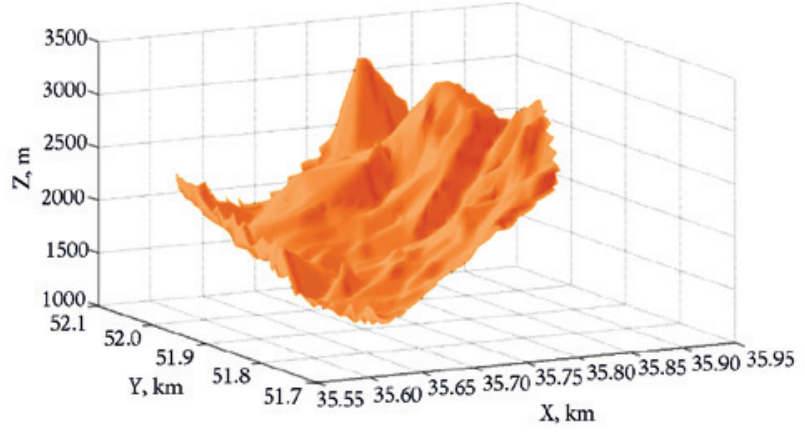

Fig. 17. Result of proposed method 
the desirable accuracy in conversion from forward coefficient to inverse ones, firstly it is better to divide the fitted cube into more altimetry layers since it increases the precision and DEM resolution quality. Secondly, by increasing the number of virtual control points, the error rate would decrease significantly. Furthermore, the running tests made in the area of the space intersection one using rational coefficients showed that while the initial value is going to be greater than the actual value, the accuracy and speed of convergence will be decreased. Although a reasonable accuracy can be achieved by placing an initial value equal to the average height of imagery area. Moreover, although the stopping criterion is very effective and would decrease the error rate, but it does not significantly affect when $\mathrm{a} \geq 4$. Besides, it is presented by the comparisons of space intersection, inverse and direct RPCs that the inverse RPCs are more practical since they do not require any precise initial values and also holding less RMSE rather than direct ones, although their data processing speed are less than the direct ones.

Here, it is being considered to automatically determine the optimum number of altimetry layers and the number of virtual controllers' points. As the use of high resolution 3D model of a specific Earth region is increasing day by day especially by the geoscientists to study the necessary environmental components and urban purposes, this can open up a new opportunity to analyze and improve the previous studies related to RPCs.

\section{References}

Abedini, A.; Najibi, N. 2010. Analyzing and simulation of underwater digital terrain model (UDTM) using airborne LiDAR hydrography (ALH) technique for hydrological and floods risks assessment, in Proceedings the Prairie Summit: 29. University of Regina, Canada.

Aguilar, M. A.; Aguilar, F. J.; Agüera, F. 2008. Assessing geometric reliability of corrected images from very high resolution satellites, Photogrammetric Engineering \& Remote Sensing 74(12): 1551-1560.

http://dx.doi.org/10.14358/PERS.74.12.1551

Aguilar, M. A.; Aguilar, F. J.; Aguer, F.; Sánchez, J. A. 2007. Geometric accuracy assessment of QuickBird basic imagery using different operational approaches, Photogrammetric Engineering and Remote Sensing 73(12): 1321-1332. http://dx.doi.org/10.14358/PERS.73.12.1321

Baltsavias, E. P.; Pateraki, M. N.; Zhang, L.; Baltsavias, E. P. 2001. Radiometric and geometric evaluation of Ikonos GEO images and their use for 3D building modeling, in Proc. of Joint ISPRS Workshop on High Resolution Mapping from Space, 19-21 September 2001. Hannover, Germany, 1-21.

Chen, L.; Teo, T.; Liu, C. 2006. The geometrical comparisons of RSM and RFM for FORMOSAT-2 satellite images, Photogrammetric Engineering and Remote Sensing 72(5): 573-579. http://dx.doi.org/10.14358/PERS.72.5.573
Cox, S. 2006. Observations and measurements, Open Geospatial Consortium Best Practices Document. Open Geospatial Consortium.

Dowman, I.; Dolloff, J. T. 2000. An evaluation of rational functions for photogrammetric restitution, International Archives of Photogrammetry and Remote Sensing 33(B3/1; Part 3): 254-266.

Grodechi, J.; Dial, G. 2003. Block adjustment of high-resolution satellite images described by rational polynomials, Photogrammetric Engineering and Remote Sensing 69(1): 59-68. http://dx.doi.org/10.14358/PERS.69.1.59

Habib, A.; Kim, K.; Shin, S.; Kim, C.; Bang, K.; Kim, E.; Lee, D. 2007. Comprehensive analysis of sensor modeling alternatives for high-resolution imaging satellites, Photogrammetric Engineering and Remote Sensing 73(11): 1241-1251. http:// dx.doi.org/10.14358/PERS.73.11.1241

Li, R. X.; Niu, X. T.; Liu, C.; Wu, B.; Deshpande, S. 2009. Impact of imaging geometry on $3 \mathrm{D}$ geo-positioning accuracy of stereo IKONOS imagery,Photogrammetric Engineering and Remote Sensing 75(9): 1119-1125. http://dx.doi.org/10.14358/PERS.75.9.1119

Lin, X.; Yuan, X. 2008. Improvement of the stability solving rational polynomial coefficients, the International Archives of the Photogrammetry, Remote Sensing and Spatial Information Sciences, Beijing 37.

Madani, M. 1999. Real-time sensor-independent positioning by rational functions, in Proceedings of ISPRS Workshop on Direct versus Indirect Methods of Sensor Orientation, 25-26 November 1999, Barcelona, Spain, 64-75.

Najibi, N.; Abedini, A.; Najibi, H. 2013. Analysis of sea ice leads in Baffin Island sea using spaced based infrared remote sensing data and mathematical hydrological models, International Journal of Geosciences Research (IJGR) 1(1): 1-11.

Najibi, N.; Abedini, A. 2013. A new approach to update urban digital maps using high resolution satellite images and GIS tools (case study: Beijing city), Earth Science India Journal 6(2): 62-69.

Najibi, N.; Arabsheibani, R. 2013. Snow-covered surface variability and DEM generation using aerial photogrammetry in Mount Odin, Canada, Geodesy and Cartography 39(3): 113-120. http://dx.doi.org/10.3846/20296991.2013.823704

Najibi, N.; Jin, S. 2013. Physical reflectivity and polarization characteristics for snow and ice-covered surfaces interacting with GPS signals, Remote Sensing 5(8): 4006-4030. http://dx.doi.org/10.3390/rs5084006

Neumaier, A. 1998. Solving ill-conditioned and singular linear systems: a tutorial on regularization, Survey Review 40(3): 636-666.

Samadzadegan, F.; Azizi, A.; Abootalebi, A. 2005. Automatic determination of the optimum generic sensor model based on genetic algorithm concepts, Photogrammetric Engineering \& Remote Sensing 71(3): 277-288.

http://dx.doi.org/10.14358/PERS.71.3.277

Sarabandi, P.; Kiremidjian, A. S.; Eguchi, R. T.; Adams, B. J. 2008. Building inventory compilation for disaster management: Application of remote sensing and statistical modeling, MCEER.

Sohn, H. G.; Park, C.H.; Chang, H. 2005. Rational function model-based image matching for digital elevation models, The Photogrammetric Record 20(112): 366-383. http://dx.doi.org/10.1111/j.1477-9730.2005.00328.x 
Tao, C. V.; Hu, Y. 2001. A comprehensive study of the rational function model for photogrammetric processing, Photogrammetric Engineering and Remote Sensing 67(12): 13471358.

Tao, C. V.; Hu, Y. 2002. 3D reconstruction methods based on the rational function model, Photogrammetric Engineering and Remote Sensing 68(7): 705-714.

Tao, C. V.; Hu, Y.; Jiang, W. 2004. Photogrammetric exploitation of IKONOS imagery for mapping applications, International Journal of Remote Sensing 25(14): 2833-2853. http://dx.doi.org/10.1080/01431160310001618392

Wang, T.; Zhang, G.; Li, D.; Tang, X.; Jiang, Y.; Pan, H.; Zhu, X.; Fang, C. 2014. Geometric accuracy validation for ZY-3 satellite imagery, IEEE Geoscience and Remote Sensing Letters, 11(6): 1168-1171. http://dx.doi.org/10.1109/LGRS.2013.2288918

Yang, X. 2000. Accuracy of rational function approximation in photogrammetry, in Proc. of ASPRS Annual Conference, Washington DC.

Zhang, L.; He, X.; Balz, T.; Wei, X.; Liao, M. 2011. Rational function modeling for spaceborne SAR datasets, ISPRS Journal of Photogrammetry and Remote Sensing 66(1): 133-145. http://dx.doi.org/10.1016/j.isprsjprs.2010.10.007
Sorosh JAHANDIDEH. M.Sc. in Photogrammetry, Faculty of Engineering, University of Tehran, Iran, E-mail: sjahandideh@ ut.ac.ir. B.Sc. in Surveying and Geomatics Engineering, Faculty of Engineering, University of Tehran, Tehran, Iran, Research interests: 3D Reconstruction, Image Processing, Point Cloud Registration, Point Cloud Matching.

Ali AZIZI. PhD in Surveying and Geomatics Engineering, Glasgow, Scotland, UK, B.Sc. in Cartography, University of Tehran, Tehran, Iran. Research interests: Digital Photogrammetry and Remote Sensing, DEM, Sensors, Environment Assessment, Aerial Photogrammetry.

Nasser NAJIBI. M.Sc. in GNSS and Remote Sensing, University of Chinese Academy of Sciences, Shanghai Astronomical Observatory, Chinese Academy of Sciences, E-mail: nsr.najibi@ gmail.com. B.Sc. (with honour) in Surveying and Geomatics Engineering, Faculty of Engineering, University of Tehran. Research interests: Hydrology and Earth Systems Sciences, Water Resource Management, Remote Sensing, GNSS-Reflectometry and Remote Sensing for Hydrosphere, Cryosphere and Earth's Surface Process. 